\begin{tabular}{|c|l|}
\hline Title & $\begin{array}{l}\text { Reconstruction of plasma shape and eddy current profile based on modified cauchy condition surface method in } \\
\text { merging spherical tokamak }\end{array}$ \\
\hline Author(s) & Ushiki, Tomohiko; Inomoto, Michiaki; Itagaki, Masafumi \\
\hline Citation & $\begin{array}{l}\text { Fusion engineering and design, 122, 35-41 } \\
\text { https://doi.org/40.1016/.fusengdes.2017.09.012 }\end{array}$ \\
\hline Issue Date & 2017-11 \\
\hline Doc URL & http://hdl.handle.net/2115/75988 \\
\hline Rights & $\begin{array}{l}\text { ○ 2017, Elsevier. This manuscript version is made available under the CC-BY-NC-ND 4.0 license } \\
\text { http://creativecommons.org/icenses/by-nc-nd/4.0/ }\end{array}$ \\
\hline Rights(URL) & http://creativecommons.org/icenses/by-nc-nd/4.0/ \\
\hline Type & Fusion Engineering \& Design (2017).pdf \\
\hline article (author version)
\end{tabular}

Instructions for use 


\title{
Reconstruction of Plasma Shape and Eddy Current Profile based on Modified Cauchy Condition Surface Method in Merging Spherical Tokamak
}

\author{
Tomohiko USHIKI ${ }^{1)}$, Michiaki INOMOTO ${ }^{1)}$, Masafumi ITAGAKI ${ }^{2)}$ \\ 1) Graduate School of Frontier Sciences, The University of Tokyo, 5-1-5, Kashiwanoha, Kashiwa, Chiba, \\ Japan \\ ${ }^{2)}$ Hokkaido University, Sapporo, Japan \\ (Corresponding e-mail address: ushiki@ts.t.u-tokyo.ac.jp)
}

This paper contains

15 pages of text,

2 tables

and 8 figures. 


\begin{abstract}
The spherical tokamak merging start-up is one of the central solenoid-free start-up method. For controlling the merging actively, it is essential to identify the plasma shapes during the merging process. In the present work, the modified Cauchy condition surface method is applied to demonstrate the reconstruction of the plasma boundary shapes as well as the eddy current profiles at three different representative phases in the merging start-up process (a. initial phase, b. merging phase, c. after merging). Profiles of magnetic flux and plasma boundary have been reconstructed accurately using noiseless magnetic sensor signals and reconstructed with a fairly good accuracy using sensor signals with $3 \%$ noise. The eddy current profile have been also reconstructed with a fairly accuracy.
\end{abstract}

Keywords: spherical tokamak; merging start-up; modified CCS method; plasma shape reconstruction; eddy current

\title{
1. INTRODUCTION
}

The idea to reduce the aspect ratio or the spherical tokamak (ST) concept [1] has the capability of attaining high- $\beta$ plasma, and is expected as a candidate of compact fusion reactor. In a ST reactor, central solenoid (CS) coil must be miniaturized or eliminated because of narrow space in the central region of the ST device. Thus, establishment of CS-free start-up scheme is strongly required. Various types of start-up methods, such as introductions of the electron cyclotron waves, the electron Bernstein waves [2, 3], the lower hybrid wave [4], the coaxial helicity injection [5-8], the induction by vertical field [9-11], and the merging technique [12, 13], are being developed in many experimental devices. 
The started-up ST plasma should be completely sustained by some non-inductive additional heating and the current drive methods using the RF waves and/or the neutral beam injection (NBI) [14]. Thus, in the ST plasmas initiated without using CS coils, the parameters of plasma, such as the plasma current, the density, and the temperature, should take values suitable for the RF/NBI power absorption.

The merging start-up method has the advantage of achieving high plasma temperature and density because it involves the reconnection heating and the compression processes. This has been demonstrated in the START, MAST (UKAEA) [15], TS-3, TS-4 (Univ. Tokyo) [12] in which in-vessel poloidal field (PF) coils are utilized to form the initial two STs inductively. In the UTST device (Univ. Tokyo) [13], all the PF coils are located outside the vacuum vessel and successfully initiate two STs with inductive electromotive force across the vessel wall. In each case, the plasma current is driven by the toroidal electric field generated by the swinging-down of the PF coil currents. In order to achieve optimal merging conditions, the initial two ST plasmas should have an identical plasma current and shape, and then move symmetrically toward the center of the device with appropriate velocity. This merging process is caused by the repulsion forces between the plasma current and the reversed PF coil currents. However, the PF coil current waveforms are provided by the LC-circuits or the pre-programmed power supplies in the present experiments. Thus, one requires an active feedback control of the plasma currents, the positions, and the shapes of the two initial ST plasmas to optimize the merging process for achieving higher plasma parameters.

To realize the active feedback control and optimize the plasma parameters described above, the shape of the last closed flux surface (LCFS) or the plasma boundary shape is highly important. Such information should be deduced from signals of magnetic sensors located near the vacuum vessel wall, since the direct measurement of physical quantities inside the plasma is usually difficult. The Cauchy condition surface (CCS) method [16-23] is one such idea for reconstructing the magnetic flux profile outside the plasma and hence the plasma boundary shape. The method has already been established for operating control and diagnosis of JT-60U [16], a tokamak-type device. The application of the method has been expanded to other devices such as KSTAR [17], JT-60SA [18] and QUEST [19]. Further, Itagaki et al. developed the 3D CCS method [20-22] to reconstruct 3D 
magnetic field profile outside the non-axisymmetric plasma in the LHD. In these methods, the CCS, where both the Dirichlet and the Neumann conditions are unknown, is hypothetically placed in a domain that can be supposed to be inside the plasma. The CCS plays the same role as the plasma current in causing the field outside the plasma. As a difficulty in the authors' research field, the initial STs in the merging start-up phase do not satisfy the equilibrium condition, however, it is fortunate that the CCS method is effective in the shape reconstruction without introducing any information on the MHD equilibrium.

Another difficulty that hampers the reconstruction is the effect of the large eddy currents generated on the vessel wall during the merging start-up period, since the initial two STs are formed inductively. These eddy currents should also be evaluated for a reliable reconstruction of magnetic field/flux structure. Recently, Itagaki et al. proposed an advanced method [23] for the RELAX, a reversed field pinch (RFP) device, where the eddy current effect on the vessel wall is incorporated into the original CCS method algorithm. In more detail, the eddy current term is given by a boundary integral along the vessel in the poloidal direction. The eddy current profile on the vessel is not given in advance but completely unknown before one starts the analysis. That is, the Cauchy conditions and the eddy current values on the vessel nodes are solved simultaneously using only the signals from magnetic sensors. Here, the authors give this advanced method [23] the name "the modified Cauchy condition surface method (the M-CCS method)". The authors believe that the M-CCS method is also suitable for the reconstruction of the magnetic field structure in the UTST, especially in the ST merging start-up period. In the present work, the M-CCS method is applied to demonstrate the reconstruction of the plasma boundary shapes as well as the eddy current profiles at three different representative phases of plasma merging process (a. initial phase, b. merging phase, c. after merging) in limiter configurations of the UTST device. This paper is arranged as follows. The outline of the M-CCS method is given in Sec. 2. The modelling and the calculation conditions for the present UTST analyses are described in Sec. 3. Reconstruction of the poloidal flux profiles and the plasma boundaries are reported in Sec. 4 . Section 5 describes the reconstructed results of eddy current profiles. In both section 4 and 5, the effects of sensor signal noise are also discussed. Section 6 gives the conclusion. The 
present research results demonstrate the effectiveness of the M-CCS method in the reconstruction analyses of ST merging process.

\section{METHOD}

\section{A) The modified Cauchy condition surface (M-CCS) method}

In the present study the authors adopted the M-CCS method for the reconstruction. Schematic of this method is shown in FIG. 1. The CCS and the vacuum vessel boundary are located to describe the plasma current effect and the toroidal eddy current, respectively. Then this is an 'inverse problem' that aims to infer the Dirichlet and the Neumann conditions on the CCS as well as the toroidal current on the vacuum vessel, based on the observation of the magnetic sensor signals outside the plasma. Three types of boundary integral equations to be solved are described as shown below [23].

(a) For poloidal flux sensors at points $i$

$$
\psi_{i}-W_{i}^{\psi}=\int_{\Gamma_{\mathrm{CCS}}}\left(\frac{\psi^{*}}{r} \frac{\partial \psi}{\partial n}-\frac{\psi}{r} \frac{\partial \psi^{*}}{\partial n}\right) d \Gamma+\mu_{0} \int_{\Gamma_{\mathrm{VV}}} j_{\mathrm{VV}}\left(\mathbf{r}_{\mathrm{VV}}\right) \psi^{*}\left(\mathbf{r}_{\mathrm{VV}} \rightarrow \mathbf{r}_{i}\right) d \Gamma\left(\mathbf{r}_{\mathrm{VV}}\right)
$$

(b) For magnetic field sensors at points $i$

$$
B_{i}-W_{i}^{B}=\int_{\Gamma_{\mathrm{CCS}}}\left(\frac{B^{*}}{r} \frac{\partial \psi}{\partial n}-\frac{\psi}{r} \frac{\partial B^{*}}{\partial n}\right) d \Gamma+\mu_{0} \int_{\Gamma_{\mathrm{VV}}} j_{\mathrm{VV}}\left(\mathbf{r}_{\mathrm{VV}}\right) B^{*}\left(\mathbf{r}_{\mathrm{VV}} \rightarrow \mathbf{r}_{i}\right) d \Gamma\left(\mathbf{r}_{\mathrm{VV}}\right)
$$

(c) For points $i$ on the CCS

$$
-W_{i}^{\mathrm{CCS}}+c_{i} \psi_{i}=\int_{\Gamma_{\mathrm{CCS}}}\left(\frac{\psi^{*}}{r} \frac{\partial \psi}{\partial n}-\frac{\psi}{r} \frac{\partial \psi^{*}}{\partial n}\right) d \Gamma+\mu_{0} \int_{\Gamma_{\mathrm{vV}}} j_{\mathrm{VV}}\left(\mathbf{r}_{\mathrm{VV}}\right) \psi^{*}\left(\mathbf{r}_{\mathrm{VV}} \rightarrow \mathbf{r}_{i}\right) d \Gamma\left(\mathbf{r}_{\mathrm{VV}}\right)
$$

where $\psi$ means the magnetic flux function [Wb/rad], and $c_{i}$ is a constant that depends on the local boundary geometry on the CCS [24]. In Eqs. (1)-(3), $W_{i}^{\psi}, W_{i}^{B}$ and $W_{i}^{\mathrm{CCS}}$ are the contributions of the external coil current to the point $i$. In each equation, $\psi^{*}$ is the fundamental solution which satisfies the equation

$$
-\left\{r \frac{\partial}{\partial r}\left(\frac{1}{r} \frac{\partial}{\partial r}\right)+\frac{\partial^{2}}{\partial z^{2}}\right\} \psi^{*}=r \delta(r-a) \delta(z-a),
$$


where $\delta(r-a) \delta(z-a)$ is Dirac's delta function. Equation (4) describes the axisymmetric poloidal flux function for an arbitrary field point $(r, z)$ caused by a toroidal current spike at the coordinate $(a, b)$. Then the fundamental solution $\psi^{*}$ is given by

$$
\psi^{*}=\frac{\sqrt{a r}}{\pi k}\left[\left(1-\frac{k^{2}}{2}\right) K(k)-E(k)\right],
$$

with

$$
k^{2}=\frac{4 a r}{(r+a)^{2}+(z-b)^{2}},
$$

where $K(k)$ and $E(k)$ are the complete elliptic integrals of the first and the second kind, respectively. The quantity $B^{*}\left(\mathbf{r}_{\mathrm{VV}} \rightarrow \mathbf{r}_{i}\right)$ means $B^{*}=-\mathbf{n}_{0} \cdot \nabla \psi^{*} / r$ with $\mathbf{n}_{0}$ being the assigned vector normal to the direction of the 'magnetic probe' located at the point $i$. The quantity $\mathbf{r}_{\mathrm{VV}}$ denotes an arbitrary point on the vacuum vessel. $j_{\mathrm{VV}}\left(\mathbf{r}_{\mathrm{VV}}\right)$ shows the linear density $[\mathrm{A} / \mathrm{m}]$ of the toroidal eddy current on the vacuum vessel. In each of Eqs. (1)-(3) the first term on the RHS comes from the original CCS method formulation. These parts of the boundary equations are derived from the Grad-Shafranov equation for a vacuum field [24]. The second term on the RHS of each Eqs. (1)-(3) describes the contribution of the eddy current. Once both of the boundary conditions on the CCS and the current density profile on the vacuum vessel have been solved, the magnetic flux value can be calculated at an arbitrary point outside the plasma.

Figure 1. Schematic of M-CCS method

\section{B) Boundary elements for discretization}

The CCS and the vacuum vessel boundary are discretized using a set of discontinuous quadratic boundary elements. Illustration of discontinuous quadratic boundary elements is shown in FIG. 2. A boundary element consists of mesh points and nodes. The mesh points define the shape of boundary element, while the nodes are 
points to give the physical quantities. Now a local coordinate $\xi$ is introduced which ranges from -1 to 1 . Then the geometry coordinate $(r, z)$ on a boundary element is given by

Figure 2. Illustration of the discontinuous quadratic boundary element

$$
r=\phi_{1} r_{1}+\phi_{2} r_{2}+\phi_{3} r_{3}, \quad z=\phi_{1} z_{1}+\phi_{2} z_{2}+\phi_{3} z_{3}
$$

with the interpolation functions

$$
\phi_{1}=\xi(\xi-1) / 2, \quad \phi_{2}=(1-\xi)(1+\xi) \text { and } \phi_{3}=\xi(1+\xi) / 2 .
$$

This quadratic element is convenient for arbitrary geometries and provides good approximation of physical quantities. The physical quantities $\psi, \partial \psi / \partial n$ and the eddy current $j$ are interpolated as

$$
\begin{gathered}
\psi(\zeta)=\Phi_{1}(\zeta) \psi_{1}+\Phi_{2}(\zeta) \psi_{2}+\Phi_{3}(\zeta) \psi_{3}, \\
\frac{\partial \psi}{\partial n}(\zeta)=\Phi_{1}(\zeta)\left(\frac{\partial \psi}{\partial n}\right)_{1}+\Phi_{2}(\zeta)\left(\frac{\partial \psi}{\partial n}\right)_{2}+\Phi_{3}(\zeta)\left(\frac{\partial \psi}{\partial n}\right)_{3}, \\
\text { and } j(\zeta)=\Phi_{1}(\zeta) j_{1}+\Phi_{2}(\zeta) j_{2}+\Phi_{3}(\zeta) j_{3} .
\end{gathered}
$$

Here, the interpolation functions for the physical quantities are different from the shape functions of Eq. (8), since the node points at both ends are shifted toward the inside. This type of boundary element is called the discontinuous element or the non-conforming element, as the physical quantities are not assumed to be continuous at both ends of an element. The interpolation functions adopted here have the forms

$$
\Phi_{1}(\zeta)=\frac{3}{4} \zeta\left(\frac{3}{2} \zeta-1\right), \Phi_{2}(\zeta)=\left(1-\frac{3}{2} \zeta\right)\left(1+\frac{3}{2} \zeta\right) \text { and } \Phi_{3}(\zeta)=\frac{3}{4} \zeta\left(\frac{3}{2} \zeta+1\right) .
$$

This use of the discontinuous elements is highly important to avoid the so-called "corner point" problem. If one uses ordinary continuous elements, a corner point sandwiched between two elements has two different values for the normal derivative of magnetic flux function depending on the side under consideration, as two continuous boundary elements hold one nodal point in common. To avoid this trouble, the adoption of the discontinuous 
elements is strongly recommended, especially for the D-shaped CCS that has two corner points (later shown in Fig.5(c)).

\section{C) Regularization technique}

In this way, finally, Eqs. (1)-(3) are discretized, coupled and can be expressed in a matrix equation form

$$
\mathbf{D p}=\mathbf{g} \text {. }
$$

The matrix equation (11) is solved using the singular value decomposition (SVD) technique [25] in such a way that the least square norm $\mathbf{P D p}=\mathbf{g} \mathbf{P}$ is minimized. The matrix $\mathbf{D}$ is decomposed as $\mathbf{D}=\mathbf{U} \mathbf{\Lambda} \mathbf{V}^{\mathrm{T}}$, where $\mathbf{U}$ and $\mathbf{V}^{\mathrm{T}}$ are orthogonal matrices and $\mathbf{\Lambda}$ is a diagonal matrix with positive singular values or zero components. The solution in this case is given by

$$
\mathbf{p}=\mathbf{V} \boldsymbol{\Lambda}^{-1} \mathbf{U}^{\mathrm{T}} \mathbf{g} .
$$

To cut out high frequent modes and avoid a numerical instability of the solution, the so-called truncated singular value decomposition (TSVD) technique [25] is used. In this technique, the regularized solution is given by

$$
\mathbf{p}_{k}=\mathbf{V} \boldsymbol{\Lambda}_{k}^{-1} \mathbf{U}^{\mathrm{T}} \mathbf{g} .
$$

Here $\boldsymbol{\Lambda}_{k}$ means that the singular values smaller than the component $\lambda_{k}$ in $\boldsymbol{\Lambda}$ are omitted so that the condition number [25] is not larger than a certain value.

The authors determined the optimized number of omitted singular values in such a way that the reconstructed profiles of magnetic flux and eddy current agree well with the reference solutions. This is because the aim of the present research is to seek the best analytic conditions for the reconstruction by comparing the reconstructed solutions with the reference solution that was given directly using a MHD equilibrium code. The number of cut-off singular values found in the present work can be a clue to make a future analysis for an actual operation as far as its modelling is almost the same as in the present cases. However, it is of course convenient that one can introduce an appropriate criterion to decide automatically the optimized number of cut-off singular values. Investigation of such kinds of criteria is one of the authors' future works. 


\section{ANALYTICAL SETTING}

Three representative phases (a. initial phase, b. merging phase, c. after merging) are considered to model limiter configurations in the UTST device, as a typical example of a spherical tokamak merging start-up. The vacuum vessel is regarded as axisymmetric in the toroidal direction and its cross section has a convex shape as shown in FIG. 1. The reference distributions of magnetic flux and the eddy current on the vacuum vessel were analyzed beforehand using a UTST equilibrium code. Figures 3 (a)-(c) show the reference poloidal flux profiles in these three phases. The blue heavy lines and red points show the plasma boundaries and limiter-plasma contact positions, respectively. In the following discussion, only the equilibrium field (EF) coil current effect is considered as the external coil effect in the formations of the flux distribution.

\section{A) Location of sensors and analytic nodes}

The magnetic sensor signals were also given from the reference flux profiles. The sensor locations in this test calculations are illustrated in FIG. 4 (a). One assumes 47 flux loops and 43 field sensors, i.e., a total of 90 magnetic sensors inside the vacuum vessel. The field sensors detect the poloidal magnetic field in a certain direction.

Segmentation of the conductor wall is shown in FIG. 4 (b). The convex shape of vacuum vessel is divided into 17 discontinuous quadratic boundary elements, so that the total number of nodes is 51 and the number of unknowns on vacuum vessel is the 51. Further, the CCS shapes are set differently in each merging phase. The CCS segmentations in the three phases are shown in FIG. 5. The numbers of CCSs, boundary elements and nodes are listed in TABLE. 1. In each of a. initial phase and b. merging phase two separated CCSs are set to enclose the corresponding two magnetic axes. In c. after merging, one sets a "D-shaped” CCS that is supposed to be almost 
similar to the shape of LCFS. When this method is applied for real time control of merging ST, the number of CCSs has to be changed from one to two at a certain point of time. In each phase, a total of 6 discontinuous quadratic boundary elements are used, so that the numbers of nodes and unknowns on the CCS are 18 and 36, respectively. Eventually, the total number of unknowns is 87, which is smaller than a total of 90 magnetic sensors. Then the uniqueness of the solution is guaranteed.

Figure 3. Reference poloidal flux profiles in initial phase, merging phase and after merging

Figure 4. Sensor locations and segmentation of the vacuum vessel wall

Figure 5. CCS segmentations at initial phase, merging phase and after merging

Table 1. The numbers of CCSs, boundary elements and nodes in each phase

\section{RECONSTRUCTED POLOIDAL FLUX PROFILE AND PLASMA BOUNDARY SHAPE}

The magnetic flux distributions and the eddy current profile were reconstructed in three different phases and the influence of sensor signal noise was studied. The Gaussian noises were numerically added to magnetic sensor signals. In the present study, one added a noise with $3 \%$ standard deviation to each sensor signal. Figures 6 (a), (b) and (c) show the reconstructed flux profiles in the three different phases. In each figure the magenta dashed contours show the reference solution, while the black solid contours indicate the reconstructed solution (noise: 
$0 \%$ ). The blue, red, green solid lines show the reference plasma boundary, the reconstructed plasma boundary (noise: $0 \%$ ) and the reconstructed plasma boundary (noise: $3 \%$ ), respectively. Further, the reconstructed poloidal magnetic field profile in each phase was shown in FIG. 7. In each figure, the abscissa denotes the distance from the "start" point that is indicated in each corresponding figure in FIG. 6, in the counterclockwise direction along the perimeter of the reference plasma boundary. The blue, red and green solid lines show the reference poloidal magnetic field profile, the reconstructed poloidal magnetic field profile (noise: $0 \%$ ) and the reconstructed poloidal magnetic field profile (noise: $3 \%$ ) on each plasma boundary, respectively. TABLE. 2 shows the number of singular values that are not truncated, the reference and the reconstructed total plasma current (Ip) in each calculation. The reconstructed Ip was calculated based on the following equation using the reconstructed plasma boundary condition

$$
I_{\mathrm{p}}=\frac{1}{\mu_{0}} \int_{\Gamma_{\mathrm{p}}}\left(\frac{1}{r} \frac{\partial \psi}{\partial n}\right) d \Gamma
$$

As shown in FIG. 6, the reconstructed flux profile in each case shows a good agreement with the reference profile except in the vicinity of $z=0$ in Phase a (initial phase). In Phase a, the two separated plasma boundaries are shown in the reconstruction, while the reference plasma has a single boundary. However, in $|z| \geq 0.1$, when the sensor noise was not added, the discrepancy of the reconstructed plasma boundary from the reference plasma boundary (in the direction normal to the reference boundary) was less than $6 \mathrm{~mm}$ in Phase a (initial phase). With $3 \%$ noise, the discrepancy in Phase a was less than $25 \mathrm{~mm}$ in $|z| \geq 0.1$.

On the other hand, in each of Phases b (merging phase) and c (after merging), the reconstructed plasma has a single boundary as the same as the reference one. When the sensor noise was not added, the discrepancy was less than $5 \mathrm{~mm}$ in Phase b and less than $3 \mathrm{~mm}$ in Phase c. When considering the sensor signal noise, the discrepancy increases as a natural result. With $3 \%$ noise, the discrepancy was less than $11 \mathrm{~mm}$ in Phase b and less than $6 \mathrm{~mm}$ in Phase c. 
The slightly large plasma boundary discrepancy in the vicinity of $z=0$ in Phase a is thought to be caused by the reference magnetic configuration itself. The plasma boundary discrepancy $\Delta r$ along the line $z=0$ can be estimated to be

$$
\Delta r=\left(\frac{\partial \psi}{\partial r}\right)^{-1} \Delta \psi
$$

That is, the plasma boundary discrepancy $\Delta r$ caused by the poloidal flux error $\Delta \psi$ increases in the region where the gradient of the poloidal flux is small. This trend causes large boundary discrepancy in the vicinity of $z=0$, however, this estimation error may not degrade the performance of the M-CCS method for the purpose to evaluate the plasma position and the current.

The poloidal magnetic field profile on the plasma boundary was reconstructed accurately in each phase, as shown in FIG. 7. Further, TABLE. 2 shows that the reference error of total Ip was under $0.08 \%, 1.2 \%$ and $0.12 \%$ respectively in Phase a, b and c even if the $3 \%$ noise was added.

Figure 6. The reconstructed poloidal flux profiles at initial phase, merging phase and after merging

Figure 7. The reconstructed poloidal magnetic field profiles along the plasma boundary at initial phase, merging phase and after merging (The abscissa denotes the distance (journey) along the perimeter of the reference plasma boundary.)

Table 2. The truncated number of singular values, reference and reconstructed Ip in each phase

\section{RECONSTRUCTED EDDY CURRENT PROFILE}


The reconstructed eddy current profiles in the three phases are shown in FIG. 8. The blue, red, green solid lines show the reference eddy current profiles, the reconstructed eddy current profiles (noise: $0 \%$ ) and the reconstructed eddy current profiles (noise: $3 \%$ ), respectively. In the present study a thin wall approximation was assumed, so that the unit of eddy current density is described as [MA/m]. Symbols of A, B C and D in FIG. 8 show the positions on the vacuum vessel that are previously indicated in FIG. 3. In each phase, when the noise was not added, the eddy current profile on vacuum vessel was reconstructed with fairly good accuracy. When the $3 \%$ noise was added, the reconstructed eddy current profiles became inaccurate, however the results roughly reproduce the whole profiles.

Figure 8. The reconstructed eddy current profiles on the vacuum vessel at initial phase, merging phase and after merging (The abscissa denotes the distance (journey) along the perimeter of the vacuum vessel boundary. Symbols A, B, C, and D show the positions of the vacuum vessel corners that are indicated in FIG. 3.)

\section{CONCLUSION AND FUTURE REMARKS}

The M-CCS method has been applied to demonstrate the reconstruction of plasma boundary shape as well as the eddy current profile for three different phases of plasma merging process (a. initial phase, b. merging phase, c. after merging) in limiter configurations of the UTST device. In the analysis the quadratic boundary elements were employed to model the complex shapes of vacuum vessel and the CCS.

The plasma boundary shape has been reconstructed accurately using noiseless sensor signals, reconstructed with fairly good accuracy using sensor signals with $3 \%$ noise. The eddy current profile has been also reconstructed with fairly good accuracy.

The test calculations are limited to only three representative cases in the UTST device. The authors applied the M-CCS method on the cases that the reference plasma shapes are symmetry with respect to the mid plane $(\mathrm{Z}=0)$. Although the M-CCS method provided asymmetric results from the sensor data with noise contamination, further investigation is required to apply this method to asymmetric reference cases. They also have a plan to install 
magnetic sensors in the actual device to realize real time control of plasma shape and location as well as of the plasma current in the merging STs. The present results using 47 flux loops and 43 field sensors can be used to determine what the sufficient number of sensors is for the actual device. They will develop the control scheme of plasma currents and axial positions by using the external PF coils to establish balanced merging condition at first, and then we will optimize the shapes of the merging plasma and the merging speed to achieve effective condition to produce high temperature and density plasma via merging process.

They believe that this analytical technique is also useful for other axial plasma merging experiments. For example, in the doublet-shaped tokamak such as the doublet III (General Atomics) [26] and the TCV (SPC) [27], merging of two droplet-shaped plasma is regarded as the preferred scenario for forming the configuration. The present technique can be also expected as an effective analytical tool for such a doublet-shaped configuration.

\section{Acknowledgments}

This work was supported by JSPS A3 Foresight Program “Innovative Tokamak Plasma Startup and Current Drive in Spherical Torus”, Giant-in Aid for Scientific Research (KAKENHI) 16K14525, 15H05750, 15K14279, 26287143, 25820434, and the NIFS Collaboration Research program(NIFS14KNWP004, NIFS16KOAP030).

\section{References}

[1] Y. -K. M. Peng, The physics of spherical torus plasmas, Phys. Plasmas 7 (2000) 1681-1692

[2] V. F. Shevchenko, M. R. O’Brien, D. Taylor, A. N. Saveliev, and MAST team, Nucl. Fusion 50 (2010) 022004

[3] M. Uchida, T. Yoshinaga, H. Tanaka, and T. Maekawa, Phys. Rev. Lett 104 (2010) 065001 
[4] T. Shinya, Y. Takase, S. Yajima, C. Moeller, H. Yamazaki, N. Tsujii, et al., Nucl. Fusion 57 (2016) 036006

[5] R. Raman, B. A. Nelson, M. G. Bell, T. R. Jarboe, D. Mueller, T. Bigelow, et al., Phys. Rev. Lett 97 (2006) 175002

[6] M. Nagata, T. Kanki, N. Fukumoto, and T. Uyama, Physics of Plasmas 10 (2003) 2932-2939

[7] A. J. Redd, T. R. Jarboe, W. T. Hamp, B. A. Nelson, R. G. O’Neill, and R. J. Smith, Physics of Plasmas 15 (2008) 022506

[8] D. J. Battaglia, M. W. Bongard, R. J. Fonck, A. J. Redd, and A. C. Sontag, Phys. Rev. Lett 102, (2009) 225003

[9] O. Mitarai, Plasma Phys. Control. Fusion 41 (1999) 1469-1483.

[10] S. Shiraiwa, S. Ide, S.Itoh, O. Mitarai, O. Naito, T. Ozeki, et al., Phys. Rev. Lett 92 (2003) 035001

[11] J. Kim, W. Choe, and M. Ono, Plasma Phys. Control. Fusion 46 (2004) 1647-1657

[12] Y. Ono, T. Kimura, E. Kawamori, Y. Murata, S. Miyazaki, Y. Ueda, et al., Nucl. Fusion 43 (2003) 789-794

[13] M. Inomoto, T.G. Watanabe, K. Gi, K. Yamasaki, S. Kamio, R. Imazawa, et al., Nucl. Fusion 95 (2015) 033013

[14] M. Ushigome, S. Ide, S. Itoh, E. Jotaki, O. Mitarai, S. Shiraiwa, et al., Nucl. Fusion 46 (2006) 207-213

[15] I. T. Chapman, J. Adamek, R. J. Akers, S. Allan, L. Appel, O. Asunta, et al., Nucl. Fusion 55 (2015) 104008

[16] K. Kurihara, Fusion Eng. Des 51-52 (2000) 1049-1057

[17] Y. Miyata, S. H. Hahn, T. Suzuki, S. Ide, J. Chung, J. G. Bak, et al., Physics of Plasmas 21 (2016) 032502

[18] Y. Miyata, T. Suzuki, M. Takechi, H. Urano, and S. Ide, Rev. Sci. Instrum. 86 (2016) 073511

[19] K. Nakamura, Y. Jiang, X. L. Liu, O. Mitarai, K. Kurihara, Y. Kawamata, et al., Fusion Eng. Des 86 (2011) 1080-1084

[20] M. Itagaki, T. Maeda, T. Ishimaru, G. Okubo, K. Watanabe, R. Seki, et al., Plasma Phys. Control. Fusion 53 (2011) 105007

[21] M. Itagaki, G. Okubo, M. Akazawa, Y. Matsumoto, K. Watanabe, R. Seki, and Y. Suzuki, Plasma Phys. Control. Fusion 54 (2012) 125003

[22] M. Itagaki, K. Ishimaru, Y. Matsumoto, K. Watanabe, R. Seki, and Y. Suzuki, Plasma Fusion Res 8 (2013) 1402134

[23] M. Itagaki, A. Sanpei, S. Masamune, and K. Watanabe, Plasma Fusion Res 9 (2014) 1402046

[24] M. Itagaki, J. Kamisawada, S. Oikawa, Nucl. Fusion 44 (2004) 427-437

[25] P. C. Hansen, Rank-Deficient and Discrete Ill-Posed Problems - Numerical Aspects of Linear Inversion (Philadelphia, SIAM, 1998).

[26] J. C. Wesley, T. Angel, C. J. Armentrout, D. R. Baker, F. P. Blau, G. Bramson, et al., in Plasma Physics and Controlled Nuclear Fusion Research (Proc. 8th Int. Conf. Brussels, 1980) Vol. 1, IAEA, Vienna (1981) 35

[27] F. Piras, EPFL Thesis 5015 (2011) 


\section{Table Captions}

Table 1. The numbers of CCSs, boundary elements and nodes in each phase

Table 2. The truncated number of singular values, reference and reconstructed Ip in each phase 


\section{Figure Captions}

Figure 1. Schematic of M-CCS method

Figure 2. Illustration of the discontinuous quadratic boundary element

Figure 3. Reference poloidal flux profiles in initial phase, merging phase and after merging

Figure 4. Sensor locations and segmentation of the vacuum vessel wall

Figure 5. CCS segmentations at initial phase, merging phase and after merging

Figure 6. The reconstructed poloidal flux profiles at initial phase, merging phase and after merging

Figure 7. The reconstructed poloidal magnetic field profiles along the plasma boundary at initial phase, merging phase and after merging (The abscissa denotes the distance (journey) along the perimeter of the reference plasma boundary.)

Figure 8. The reconstructed eddy current profiles on the vacuum vessel at merging initial phase, merging phase and after merging (The abscissa denotes the distance (journey) along the perimeter of the vacuum vessel boundary. Symbols A, B, C, and D show the positions of the vacuum vessel corners that are indicated in FIG. 3.)

\begin{tabular}{|c|c|c|c|}
\hline phase & No. of CCSs & No. of boundary elements & No. of nodes \\
\hline (a) initial phase & 2 & $3 \times 2$ & 18 \\
\hline
\end{tabular}




\begin{tabular}{|ll|c|c|c|}
\hline (b) & merging phase & 2 & $3 \times 2$ & 18 \\
\hline (c) & after merging & 1 & 6 & 18 \\
\hline
\end{tabular}

Table 1. The numbers of CCSs, boundary elements and nodes in each phase

\begin{tabular}{|c|c|c|c|c|}
\hline Phase & $\begin{array}{c}\text { Added sensor } \\
\text { noise (\%) }\end{array}$ & $\begin{array}{l}\text { No. of not truncated } \\
\text { singular values }\end{array}$ & $\begin{array}{c}\text { Reference Ip } \\
\text { (kA) }\end{array}$ & $\begin{array}{c}\text { Reconstructed Ip } \\
(\mathbf{k A})\end{array}$ \\
\hline \multirow{2}{*}{ initial phase } & 0 & 77 & \multirow{2}{*}{70.00} & 70.23 \\
\hline & 3 & 76 & & 70.52 \\
\hline \multirow{2}{*}{ merging phase } & 0 & 74 & \multirow{2}{*}{66.41} & 66.77 \\
\hline & 3 & 72 & & 67.20 \\
\hline \multirow{2}{*}{ (c) after merging } & 0 & 80 & \multirow{2}{*}{70.00} & 70.12 \\
\hline & 3 & 76 & & 70.81 \\
\hline
\end{tabular}

Table 2. The truncated number of singular values, reference and reconstructed Ip in each phase 


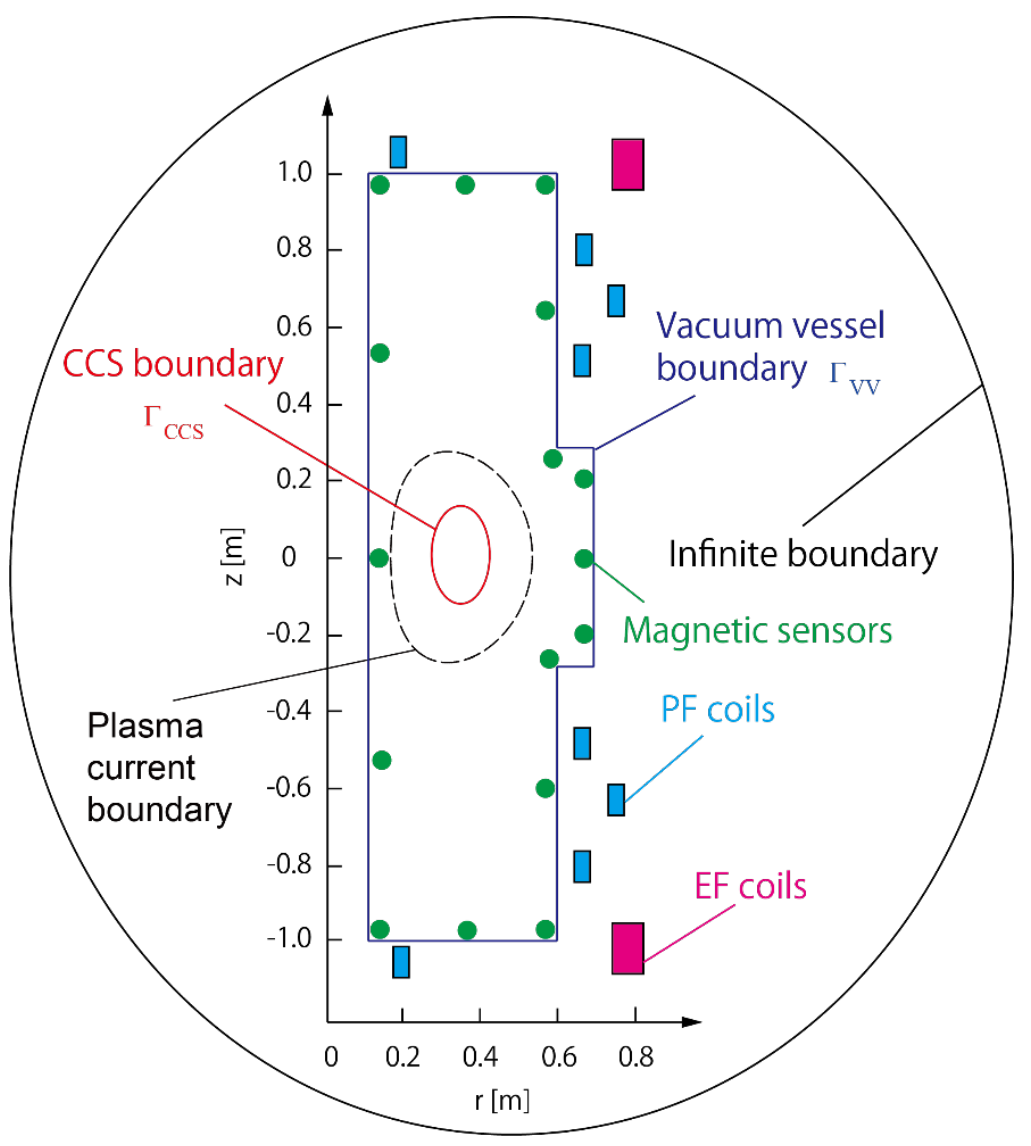

Figure 1. Schematic of improved CCS method 


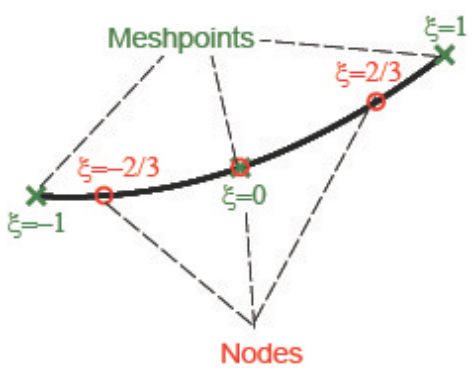

Figure 2. Illustration of discontinuous quadratic boundary elements 

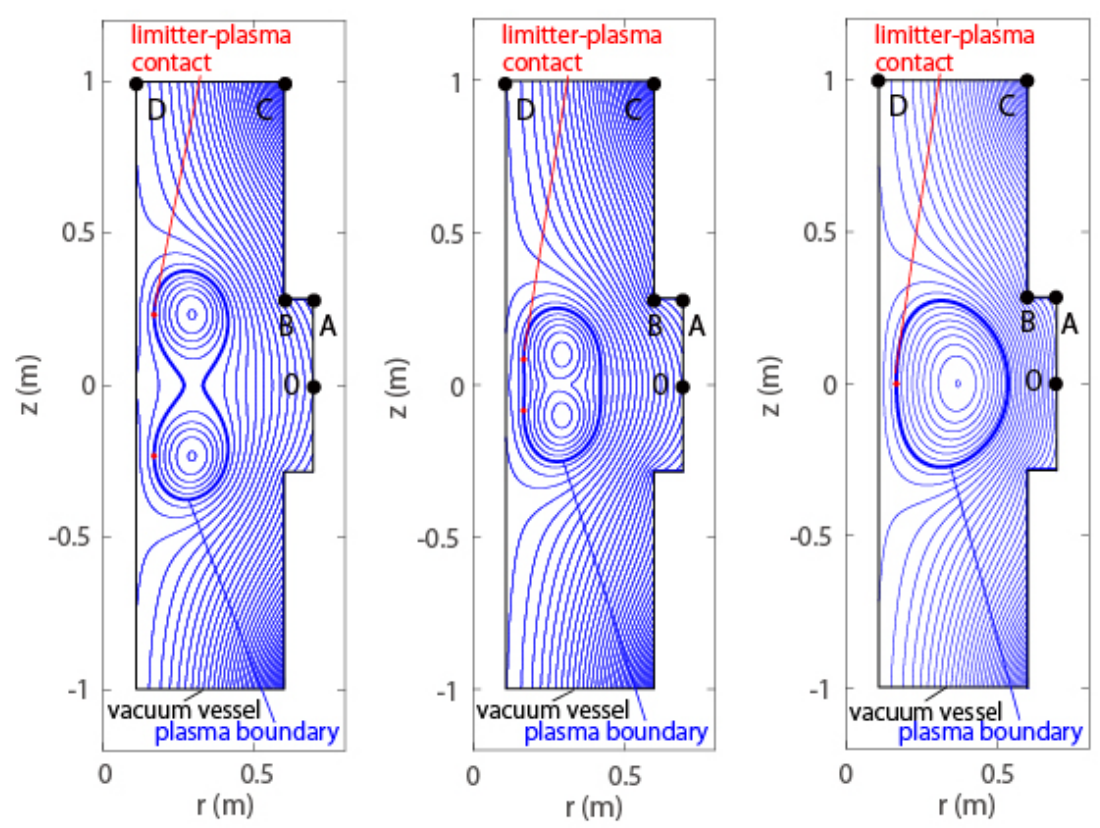
(a) initial phase
(b) merging phase
(c) after merging

Figure 3. Reference poloidal flux profile in merging initial phase, merging phase and after merging 

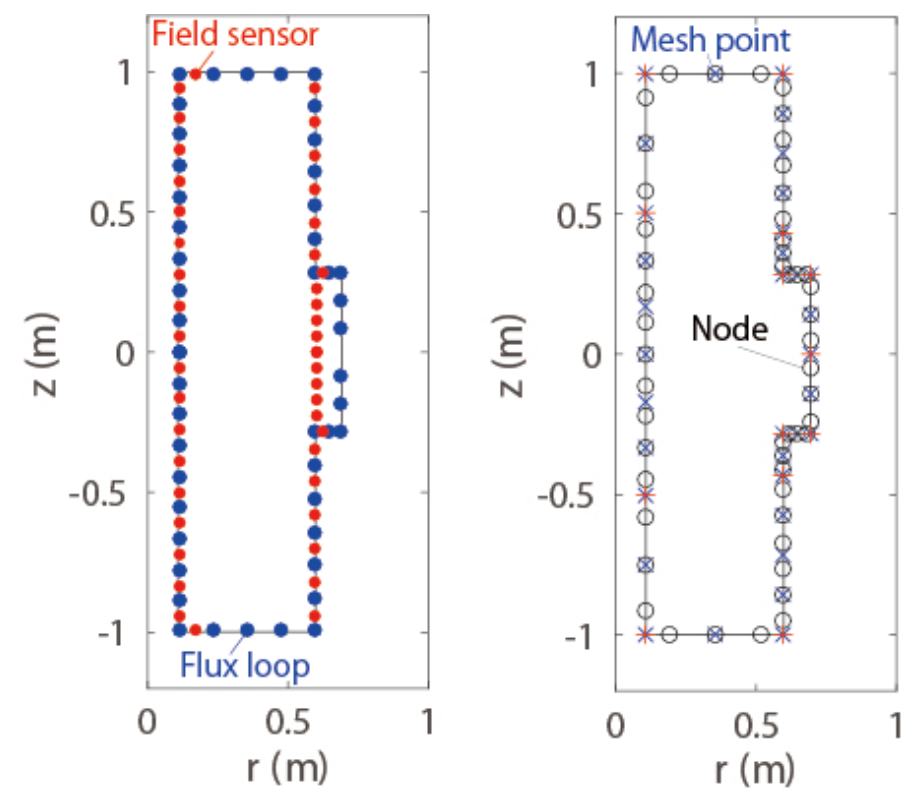

$\begin{array}{ll}\text { (a) sensor locations } & \text { (b) segmentation of the vacuum vessel wall }\end{array}$

Figure 4. Sensor locations and segmentation of the vacuum vessel wall 


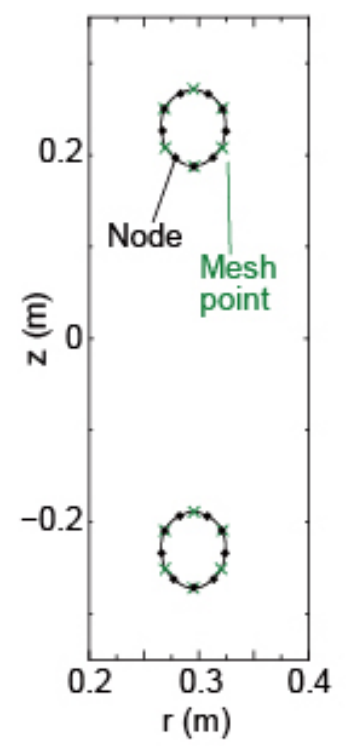

(a) initial phase

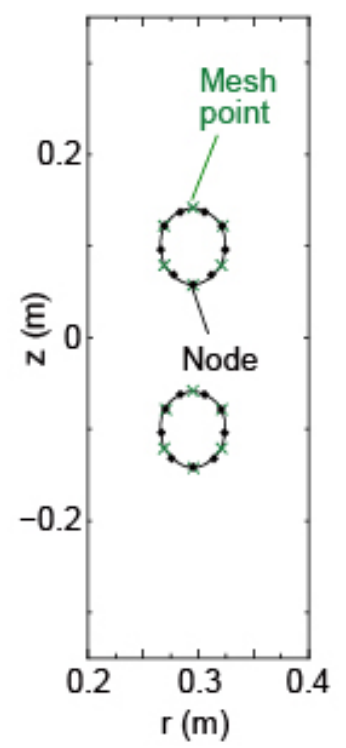

(b) merging phase

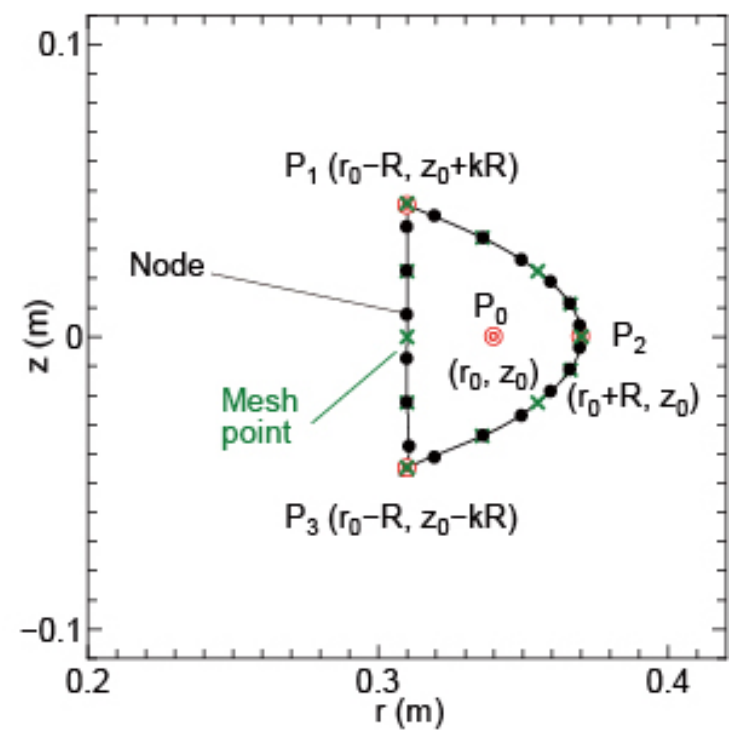

(c) after merging

Figure 5. CCS segmentations at initial phase, merging phase and after merging 


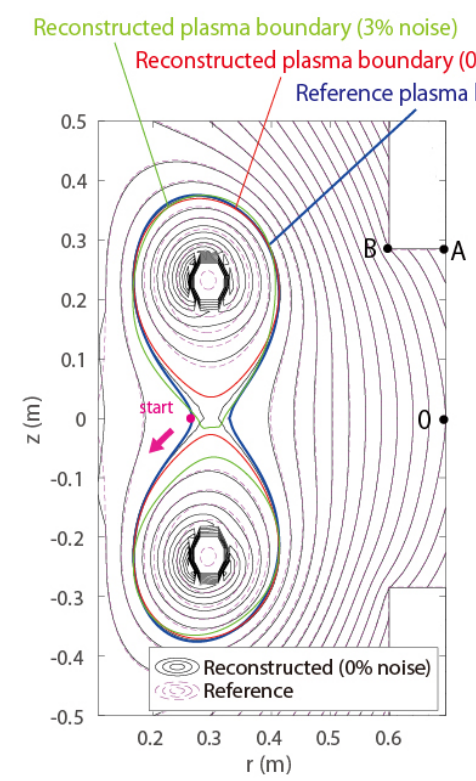

(a) initial phase

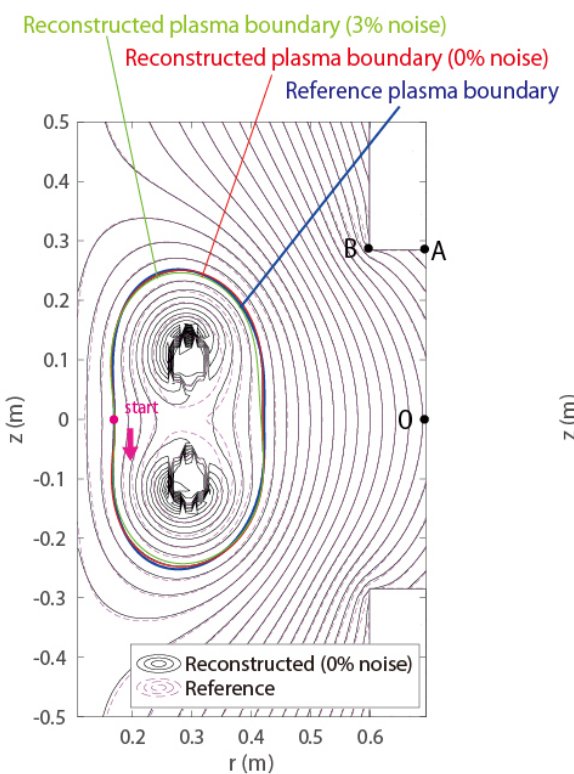

(b) merging phase

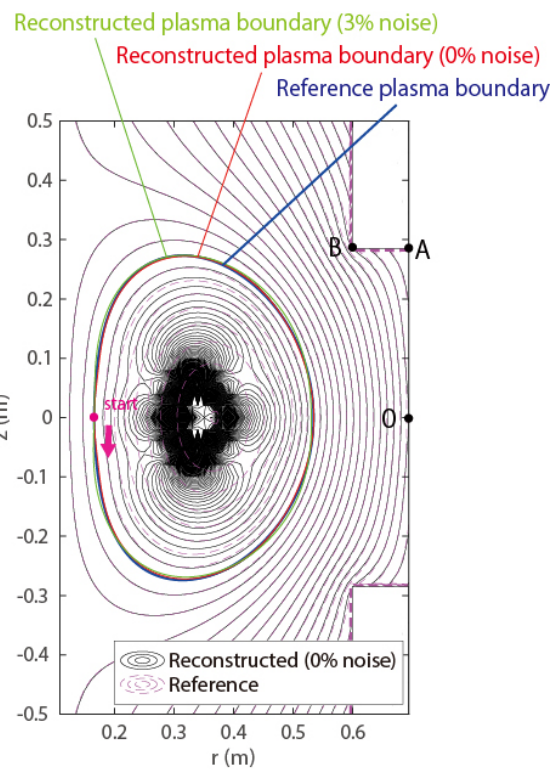

(c) after merging

Figure 6. The reconstructed poloidal flux profiles at initial phase, merging phase and after merging 


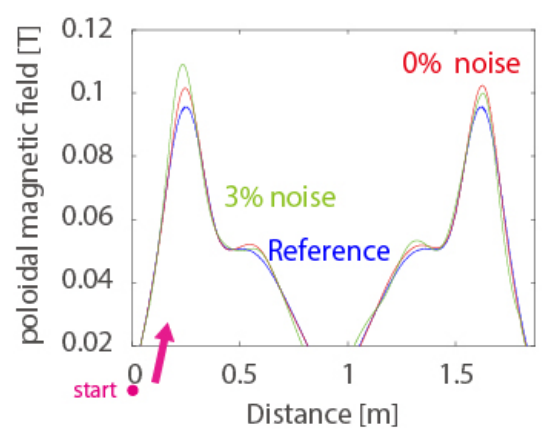

(a) initial phase

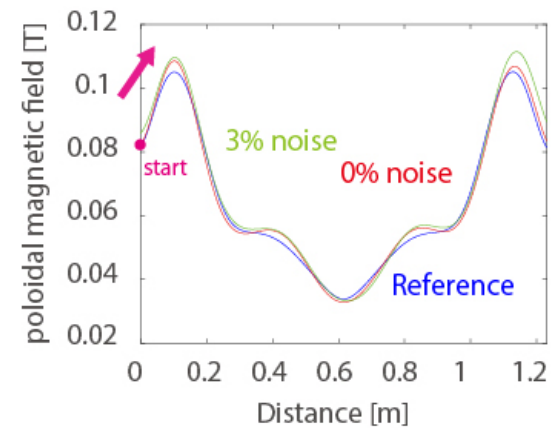

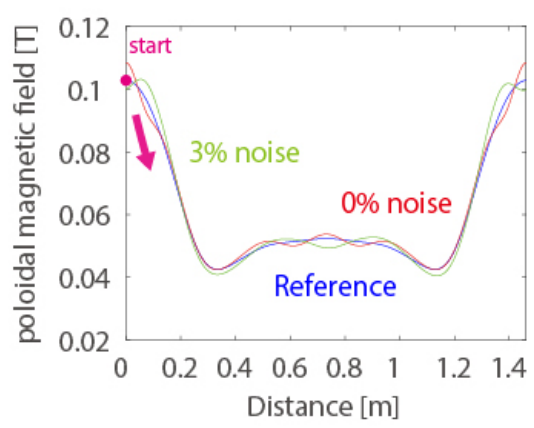

(c) after merging

Figure 7. The reconstructed poloidal magnetic field profiles along the plasma boundary at initial phase, merging phase and after merging (The abscissa denotes the distance (journey) along the perimeter of the reference plasma boundary.) 


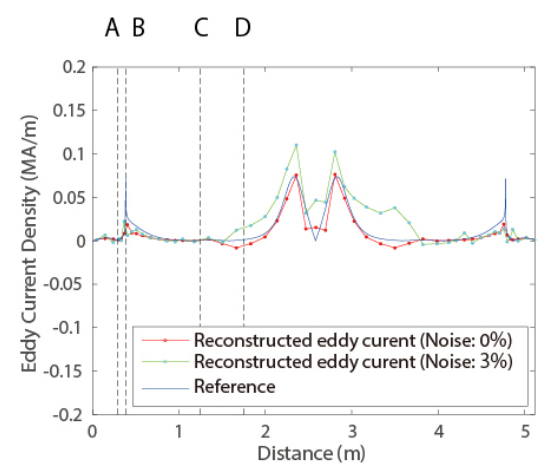

(a) initial phase
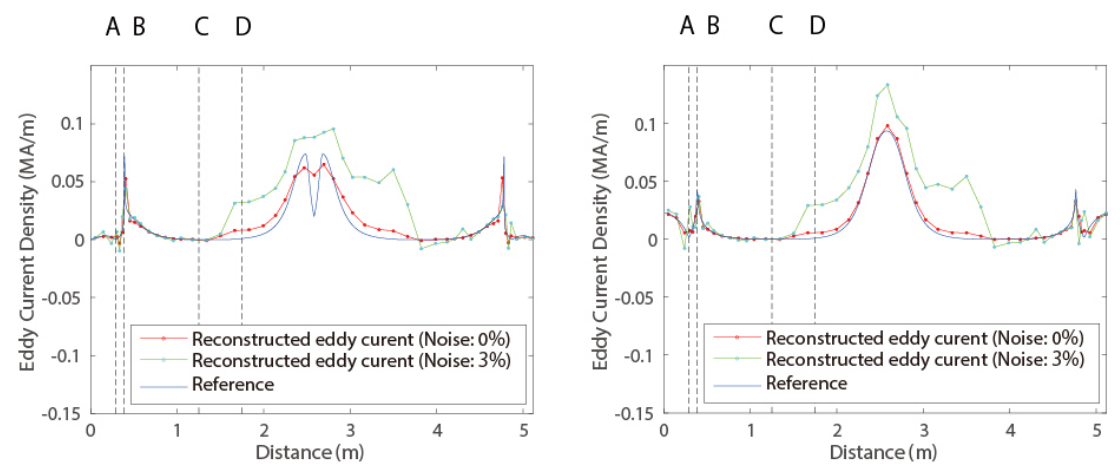

Figure 8. The reconstructed eddy current profile on the vacuum vessel at initial phase, merging phase and after merging (The abscissa denotes the distance (journey) along the perimeter of the vacuum vessel boundary.

Symbols A, B, C, and D show the positions of the vacuum vessel corners that are indicated in FIG. 3.) 